\title{
FIRST RESULTS OF PROTON INJECTION COMMISSIONING OF THE AGS BOOSTER SYNCHROTRON*
}

\author{
R.K. Reece, L. Ahrens, J. Alessi, E. Bleser, \\ J.M. Brennan, A. Luccio, J. Skelly, A. Soukas, \\ W. van Asselt, W.T. Weng, R. Witkover
}

AGS Department, Brookhaven National Laboratory Associated Universities, Inc., Upton, NY 11973 USA

\section{Summary}

Beam performance for the injection phase of protor: beam commissioning of the AGS Booster synchrotron will be presented. The beam from the $200 \mathrm{MeV}$ Linac is transported through a new beam line into the Booster. This Linac-to Booster (LTB) beam line includes a $126^{\circ}$ bend and brings the injected beam onto the Booster injection orbit through the backleg of a main ring dipole magnet. Transfer of beam from the Linac to the Booster, spiralling beam and closing the orbit in the Booster ring are discussed. Injection and transport through one sector of the ring bas been accomplished.

\section{Review of Booster Facilities}

The transfer of beam to the Booster synchrotron from the Linac includes a transport beam line of 114 feet in length. This LTB beam line is made up of three sections (Fig. 1). Section 1 runs from the deflection point in the

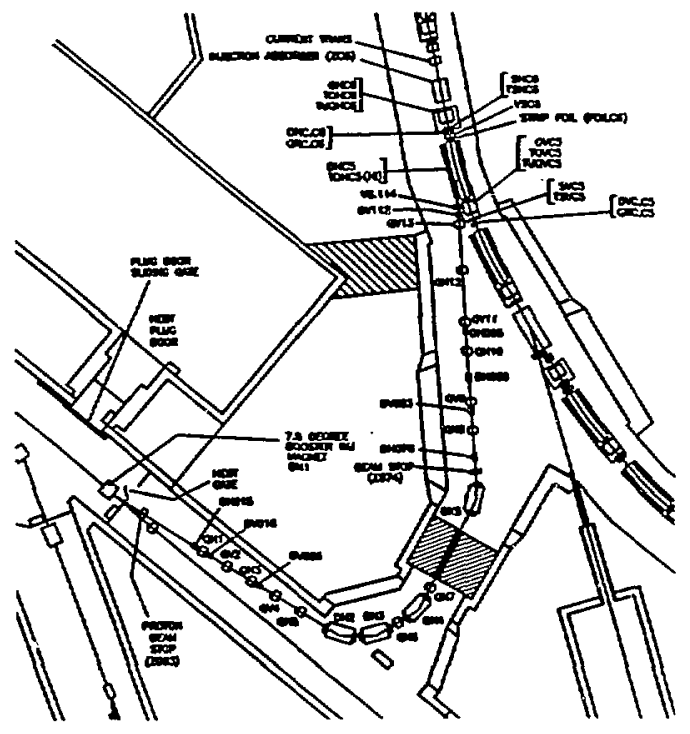

Fig. 1. LTB beam line and injection region.

-Work performed under the auspices of the U.S. Department of Energy.
Linac High Energy Beam Transport (HEBT) beam line (that transports beam to the AGS) to the $126^{\circ}$ bend region. Section 2 is the $126^{\circ}$ bend region from $\mathrm{DH} 2$ to DH5. Section 3 runs from the exit of DH5 into the Booster through the backleg iron of the $\mathrm{C5}$ main ring dipole.

The LTB section 2 is an achromatic bend region that spans the transition shielding between the HEBT area and the Booster ring enclosure. The $126^{\circ}$ bend is created with four identical dipoles powered in series and has two quadrupoles. Section 1 contains five quadrupoles and Section 3 contains six quadrupoles to permit the beam to be optically matched at the entrance to the bend region and into the Booster zing respectively.

The Booster ring[1] has a FODO lattice with missing main dipole magnets to allow for injection/extraction magnets, RF cavities and other insertions. There are forty-eight half-cells with 24 quadrupoles in each plane. The main dipoles and quadrupoles are powered in series. The main quadrupoles have additional primary and secondary windings for tune and stopband ccrrection respectively. There are also correction dipole magnet assemblies at each main quadrupole magnet tor orbit correction and local orbit bumps in both planes.

\section{Instrumentation}

Detailed descriptions of the beam instrumentation deveioped for use in the Booster facility can be found elsewhere in these proceedings. However, an overview of their application is included.

\section{Linac-To-Booster (LTB) Instrumentation}

There are two beam current transformers, one at each end near the HEBT beam line and near the Booster ring.[2]

The beam position measurement in this line is done by using seven resonant stripline beam position monitors, each providing both horizontal and vertical information. [3] In each monitor, the four plate sum signal provides in addition an approximate beam current measurement.

Beam profile information in LTB is obtained using two insertable multiwire profile monitors. The data provided by these monitors in conjunction with a sequence 


\section{DISCLAIMER}

This report was prepared as an account of work sponsored by an agency of the United States Government. Neither the United States Government nor any agency thereof, nor any of their employees, makes any warranty, express or implied, or assumes any legal liability or responsibility for the accuracy, completeness, or usefulness of any information, apparatus, product, or process disclosed, or represents that its use would not infringe privately owned rights. Reference herein to any specific commercial product, process, or service by trade aame, trademark, manufacturer, or otherwise does not necessarily constitute or imply its endorsement, recommendation, or favoring by the United States Government or any agency thereof. The views and opinions of authors expressed herein do not necessarily state or reflect those of the United States Government or any agency thereof. 
of programmed changes in specific LTB quadrupole magnets settings, can yield for the user an emitiance and Twiss parameter measurement[4] for a specified location in the beam line.

Beam loss information[5] along the beam line is given by eight coaxial loss monitor cables distributed in a contiguous manner throughout LTB. This loss monitor system is a nearly generic module in the overall loss monitor system for the Booster facility.

\section{Booster Ring Instrumentation}

The first instrumentation element the incoming proton beam intercepts in the Booster ring after being stripped to $\mathrm{H}^{+}$in a carbon foil is one of 46 split plate Pick Up Electrodes (PUE's).[6] These PUE's each measure beam centroid position in one plane and are located immediately prior to each quadrupole magnet that focuses the beam in the plane of measurement. There are 24 in the vertical plane and 22 in the horizontal; one horizontal PUE is missing at the extraction point and one missing at the Internal Beam Dump because of the horizontally oversized vacuum chambers at these locations.

Just past the immediate proton injection point is located a current transformer assembly that has incorporated in it two transformers. The Injection Transformer has a time constant capable of monitoring the injected beam pulse from the Linac of 150 to $250 \mu \mathrm{s}$ and the other Circulating Beam Transformer with an appropriate time constant to monitor the remainder of the acceleration cycle, ( $\sim 60 \mathrm{~ms}$ for protons).

The current transformers and the PUE's respond to both stripped and unstripped beams (with the appropriate sign reversal). Remote control of the stripping foil position (in/out) allowed a very valuable switching between these conditions.

\section{Preparation for Commissioning}

Before any part of the Booster facility could be commissioned with beam and in addition to the installation and testing activities, each phase of commissioning had to be prepared and thoroughly reviewed. In particular, all new beam areas are considered from the point of view of the creation of new radiation sources. As part of the process there had to be included an outline for conducting the beam tests and the area(s) reviewed beforehand to identify the locations where a credible accelerator fault could produce a local beam loss with the resulting prompt radiation. These beam fault conditions then had to be created and the studies conducted as part of the commissioning procedure. The results are then reviewed before continuing with the next phase of commissioning. Also, the personnel necessary to participate in each aspect of commissioning were trained prior to carrying out the tests with beam.

\section{Commissioning with Beam}

Commissioning of the Booster facility has been done in phases as each separable area of the accelerator is completed. The phases were identified as $200 \mathrm{MeV}$ beam transport through the first two sections of the LTB beam line, transport through all of LTB and to the Booster injection region, transport through one sextant of the Booster, spiralling beam, accelerating beam and finally extracting beam from the Booster. Final stages of Booster ring equipment installation prohibited the spiralling of beam in the Booster and as of this writing, the beam has been transported through the injection beam line and around one sextant of the ring. The ability to transport beam to this point has been invaluable in providing the first beam control and measurement information from the available diagnostics.

In order to match the beam optics at the entrance to the LTB beam line, the HEBT emittance and Twiss parameters were determined at the LTB injection point using a Least Squares fit to HEBT profile data. The range of movement in any of the Twiss parameters at this point is constrained by the fact that both the AGS and the Brookhaven Linac Isotope Production (BLIP) facility are serviced by the HEBT beam line. However, the range over which these incoming beam parameters may be varied was sufficient to define the input trajectory and optical conditions and transfer the beam into the LTB beam line. Control of the LTB optics and trajectory to the Booster ring was then straightforward using magnet calibration data.

At the exit of I.TB section 2, a temporary carbon beamstop was installed to allow the transport of low intensity beam through LTB sections 1 and 2 so that beam tests could proceed with the beam aborted safely in one location and not create residual radiation in areas where the installation effort continued. This was very important in the early stages of testing the instrumentation, data acquisition and methods of controlling beam parameters, (trajectories and optics).

Beam position measurements from the stripline BPM's in the LTB beam line reproduced to better tham $0.5 \mathrm{~mm}$. The data from the BPM's was then acquired and used in a user friendly trajectory correction program to determine and implement the necessary changes in correction dipoles to minimize orbit errors in the beam line. The details of this program and associate:algorithms can be found elsewhere in these proceedings. [7]

The beam loss monitor system proved to be sensitive to beam loss of less than $1 \times 10^{10}$ protons per pulse at a given iocation. The output of the loss monitor system is coupled with the Fast Beam Interrupt (FBI) system used to provide a rapid inhibit $(-15 \mu \mathrm{s})$ of the injected Liriac beam if integrated beam loss exceeds an assigned limit. 
With the commissioning of the AGS Booster as an accelerator, new aspects of the controls system for the AGS facility have also been undergoing a commissioning process. Included in this is the first use of a timing system environment that is centered around the ability to operate each accelerator (Linac, Booster and AGS) for muitiple users in a Pulse to Pulse Modulated (PPM) manner. This tool has been used at the PS at CERN for many years to increase the available access to the beam in individual accelerators within the facility. Each software utility developed now has the capability to acquire data for any or all separate users of the beam. One of the new software tools that has proven to be very useful in providing the user with real-time machine data (as a function of PPM beam user) is the General Purpose Monitor (GPM).[8] In this program, the user is able to acquire and display data from any defined collection of devices as a function of time. It is also "user friendly" in that the association of devices of interest is trivially modified (even amid the standard commissioning chaos) for immediate display. This has been used extensively for radiation monitoring.

When the proton injection area of the Buoster ring was available to accept beam, one sextant of the main ring buss (dipoles and quadrupoles in series) was powered by an independent DC power supply so as to assure that the beam could not be carried beyond a well defined location in the ring. Beam was then transported through section 3 of the LTB beam line and injected into the Booster ring at the $\mathrm{CS}$ location.

There was some concern over the beam trajectory through the $\mathrm{C} 5$ main dipole magnet. The incoming $\mathrm{H}^{-}$ beam from the LTB line initially traverses a field free region of the $\mathrm{C} 5$ magnet but as it crosses (at an angle of approximately $11^{\circ}$ to the tangent of the magnet) between the C5 magnet coils, the field increases quickly to the central dipole field of the magnet. This effect was studied extensively by mapping the field and model tracking of particles through this magnet. Since the net dipole effect on the incoming $H^{-}$must bring the beam to a nominal $x, x^{\prime}$ as determined by the injection orbit at this location, it was essential to know this trajectory precisely. Position measurements at the first PUE in the Booster ring (C6 quadrupole) are within estimated error of the position of the model Booster injection orbit. Angle information at the injection foil will determine whether the injected beam will have the appropriate trajectory to merge with the equilibrium orbit of the Booster ring. Model studies have shown that if this is not the case, a small translation of the downstream LTB beam line elements will be necessary and sufficient to correct any misaligament.

\section{Future Commissioning}

Installation and preliminary testing of the Booster ring equipment will be completed by the end of May. Since one of the two proton RF cavities is presently ready to be used with beam, beam spiralling and acceleration should be achieved by mid-June. The extraction equipment will be fully tested and available for beam tests also by this date. Since the AGS is running for the High Energy Physics (HEP) program, access to the AGS ring for equipment installation and testing of the Booster To AGS (BTA) transfer beam line is restricted. In order that the extraction process can be tested, another temporary beamstop has been installed a few meters into the BTA line.

All of the commissioning efforts have been and will continue to be done at a relatively low beam intensity until the machine is well understood. Higher intensity studies will follow early in FY'92.

\section{Acknowledgements}

These first commissioning achievements represent the results from the long efforts of a large community of dedicated people at BNL. In particular, we wish to recognize the mechanical and electrical engineering support by J. Brodowski and I. Marneris, respectively, the AGS Main Control Room operations personnel who helped smooth the edges at each commissioning step; Controls Group participation by D. Barton, W. Buxton, T. Clifford, B. Culwick, R. Enriquez-Leder, and V. Wong played an essential role. Finally, the wise counsel of several colleaguen, especially D. Beavis, J.W. Glenn, Y.Y. Lee, D. Lowenstein, and L. Ratner helped to make the commissioning a more coherent process.

\section{References}

1. AGS Booster Design Manual (Rev. 1, Oct., 1988).

2. R.L. Witkover, private communication.

3. T. Shea, Beam Position Monitoring in the AGS Linac-to-Booster Transfer Line, this proceedings.

4. J. Skelly, Generalized Emittance Measurements in a Beam Transport Line, this proceedings.

5. E. Beadle, The AGS Booster Beam Loss Monitor System, this proceedings.

6. D. Ciardullo, The AGS Booster Beam Position Monitor System, this proceedings.

7. T. D'Ottavio, User Control of the Proton Beam Injection Trajectories in the AGS Booster Synchrotron, this proceedings.

8. J.T. Morris, private communication. 